\title{
REMARK - Reusable Agent-Based Experience Management and Recommender Framework ${ }^{\star}$
}

\author{
Zoltan Balogh ${ }^{1}$, Michal Laclavik ${ }^{1}$, Ladislav Hluchy ${ }^{1}$, Ivana Budinska ${ }^{1}$, and \\ Krzysztof Krawczyk ${ }^{2}$ \\ 1 Institute of Informatics, SAS, Dubravska cesta 9, Bratislava 84237, Slovakia \\ \{balogh.ui, laclavik.ui, hluchy.ui, utrrbudi\}@savba.sk \\ 2 ACC CYFRONET AGH, Nawojki 11, 30-950 Cracow, Poland \\ krafcoo@icsr.agh.edu.pl
}

\begin{abstract}
In this paper we introduce an advanced experience management and recommendation framework. The framework exploits software agent technology to ensure distributed and robust functioning. In design and implementation we use state of the art methodologies, technologies and tools. Ontology is used for modeling and describing knowledge. The infrastructure is able to provide relevant recommendations to a requester entity based on recorded experiences from the past depending on the current context of the environment. Such infrastructure is suitable for environments where instant recommendation is required in a given situation. A concrete example implementation of this framework is introduced in the public administration organization where employees need knowledge according to their current work context which is represented by activities in a workflow process.
\end{abstract}

\section{Introduction}

There are many experience management (EM) frameworks but each of them is usually liaison to a concrete application area. Having a general reusable infrastructure for EM where final implementation would not require redesign of the whole system but only customization and maintenance of a knowledge model is highly demanded. In this paper we describe such experience management framework which employs software agent technology. Our aim was to make our framework integrable with existing software so we do not create a barrier for information flows.

This introduction is followed by a section in which we give an overview of relevant experience management systems and sketch the motivation of our work (Section 2). The core framework is described in Section 3. CommonKADS is a methodology for developing and maintaining knowledge management (KM) systems. We discuss the use of CommonKADS methodology for developing systems based on our framework is Section 4. A project called Pellucid 8910 being

\footnotetext{
* This work was supported by EC Project Pellucid 5FP RTD IST-2001-34519 and Slovak Scientific Grant VEGA 2/3132/23.
} 
developed in scope of the 5 th EU FP is introduced in section 5 as a verification of our framework. We conclude (in Section 6) with account of further possible implementation areas for the framework.

\section{Motivation and State of the Art}

The goal of developing an EM system is to create a knowledge repository that contains problem solving experiences for a complex problem domain that changes over time. There have been proposed several systems which we examine in this section.

HOMER [1] is a generic experience management architecture and a set of related tools that stores the experience of the help-desk operators in a case base and enable them to access, reuse, and extend the knowledge in a natural and straightforward manner. HOMER has been developed mainly for realizing helpdesk and self-service experience management applications. An object-oriented approach is used to model the experiences in HOMER. The system is implemented utilizing pure client-server architecture. Each request must be processed by the server. Server is therefore the critical point of the overall architecture what is typical for purely centralized architectures.

In 2. Lacher and Koch propose a theoretical reference agent-based knowledge management framework called Knowledge Management Information Technology (KM IT). Authors identified the advantages to employ software agents in KM system. The framework is built of agencies made up of several agents each. The agencies represent the organizational knowledge entities and manage the respective information objects. Also a so called shared ontology is proposed used for consistent communication among agencies as a basis for the user profile in the user agency and to specify information requests and replies. Even though KM IT proposes to use agents and ontologies in EM system design, the whole framework is purely theoretical with no implementation or verification work.

In 3] authors describe a development of an Intranet-based knowledge management framework called KnowledgePoint in a consulting firm. The paper further introduces a simple ontology model of organizational knowledge. The article is rather a case study than a scientific article. Anyhow very important implementation problems are raised here such as security or managerial issues in the organization. Scientists often neglect such problems whereat those must be considered during the system design.

There are also other KM systems but they usually deal only with a concrete knowledge model design and implementation. Such projects are CoMMA 4],DECOR [5], FRODO [6] or KnowMore 7].

All these above described systems address only a specific problem domain. That makes these systems too specialized and therefore inappropriate as candidates for generic EM framework. We aimed at developing a flexible and adaptable platform for enhancing EM in organizations. The framework is a set of methods, tools and reusable components that may be applied to develop further EM applications. 


\section{Framework Description}

Herein we describe the component architecture for advanced experience management. The main goal was to create a general infrastructure for EM. The infrastructure is primarily intended for use for human users, but can be easily applied to serve computational clients as well. Henceforward we will refer to any entity which is served from the framework as client. The overall framework is on the figure bellow.

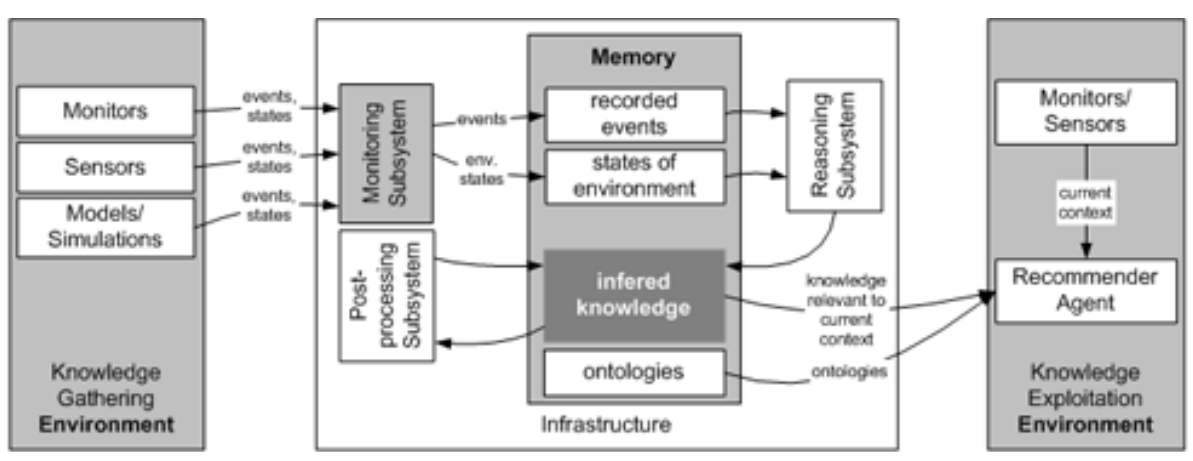

Fig. 1. The REMARK framework

Processing of knowledge in the infrastructure is fulfilled in three stages which are typical for the EM domain: capture, capitalization and reuse. In the capture phase knowledge is recorded. Additional reasoning and data mining is performed in the capitalization phase where the inferred knowledge is produced. Recorded and inferred knowledge is then used to assist users in the reuse phase. The core of the framework is the Processing Infrastructure (PI). PI has the following components:

- Corporate Memory (CM),

- Monitoring Subsystem (MSS),

- Reasoning Subsystem (RSS),

- Post-processing Subsystem (PPSS),

- Recommender Subsystem (RSS).

There are also monitors and sensors which are part of the framework but are not executed inside the PI. Monitors/sensors need to be executed on the application side with enabled active environment sensing and monitoring interfaces. Software agents have been chosen for the overall implementation of the framework. The main advantage of using agent technology is the distributed nature of agents, their well established communication infrastructure based on ontology and execution autonomy and mobility (required mainly for sensors and monitors). 
All the information stored in scope of the framework is modeled by the ontology, what is simply the object model of the real world. The ontology is extensible, what makes also the framework open. We have identify the need for:

- a Generic Ontology which is usable for any application and

- a Domain-Specific Ontology which is specific for concrete application domain.

The Knowledge Gathering Environment (KGE) was separated from the Knowledge Exploitation Environment (KEE) in the schema. KGE is simply the environment in which the knowledge is gathered. KEE is monitored and depending on the context concrete experience is recommended to the client. KGE can be but must not be identical with KEE. The knowledge is gathered from KGE by Sensor Agents (SA) or directly from a running model or simulation. SA communicates the captured information to the Monitoring Agent (MA) which records the information to the CM. The structure of information monitored by $\mathrm{SA}$ is:

- the context of selected properties of the KGE and

- the events performed in the KGE.

Both context and events must be modeled by ontology. In simpler cases context can be modeled as part of the event concept and therefore only events need to be modeled. In most cases events and context will share some common general and domain-specific concepts.

Stored contexts and events are processed by the Reasoning Agent (RE) which generates active experiences in various forms. These experiences are stored as inferred knowledge in the CM. The structure of possible recommendations presented to the client is pre-modeled. The set of pre-modeled recommendations can be extended according to application needs.

SA are used also to monitor the context of the KEE. SA communicates the monitored information to the Recommender Agent (RCA). If RCA finds knowledge which is valuable for the client in the current context it will return the knowledge to client. RCA may utilize Task Agents (TA) for concrete experience retrieval from the CM. The recommendation is handed over to Personal Assistant Agent (PAA) in a case when the client is a human user.

\section{Development Methodology}

CommonKADS [11] methodology is standard for developing knowledge management systems and can be very well used for creating model for our experience management framework. It contains of several standard model describing a problem domain:

- Organizational Model, which describes organization structure, its goals, and also problem domain of concrete application;

- Task Model describes tasks preformed in organization; 
- Agent Model describes actors involved in organization and it can be humans or computer systems involved in performing tasks;

- Knowledge and Communication Model are created from 3 models above and describes knowledge and communication between actors and

- Design Model comes from Knowledge and Communication model and describes how system should be implemented.

Knowledge model is basically ontology which describes entities (users, problems, application/domain related terms, resources) and its relation in the organization. In our framework we utilize DAML+OIL 15] based on XML/RDF for interfacing with existing systems and also for processing of knowledge results.

\section{Framework Implementation}

The described framework was verified in the Pellucid (IST-2001-34519) EU project [8]. The aim of the Pellucid platform is to provide assistance for organizationally mobile employees. Capture and capitalization of employee's knowledge in public organizations was the main interest in the project. Workflow process is used as a work context for knowledge creation, capitalization and reuse. It means that each event or action performed in an organization is stored in respect to the current workflow process status. Stored and capitalized knowledge is then recommended to other employees as they work on particular tasks in the process.

Knowledge ontology was created according to the application. First we have created an Organizational Model (Fig 2).

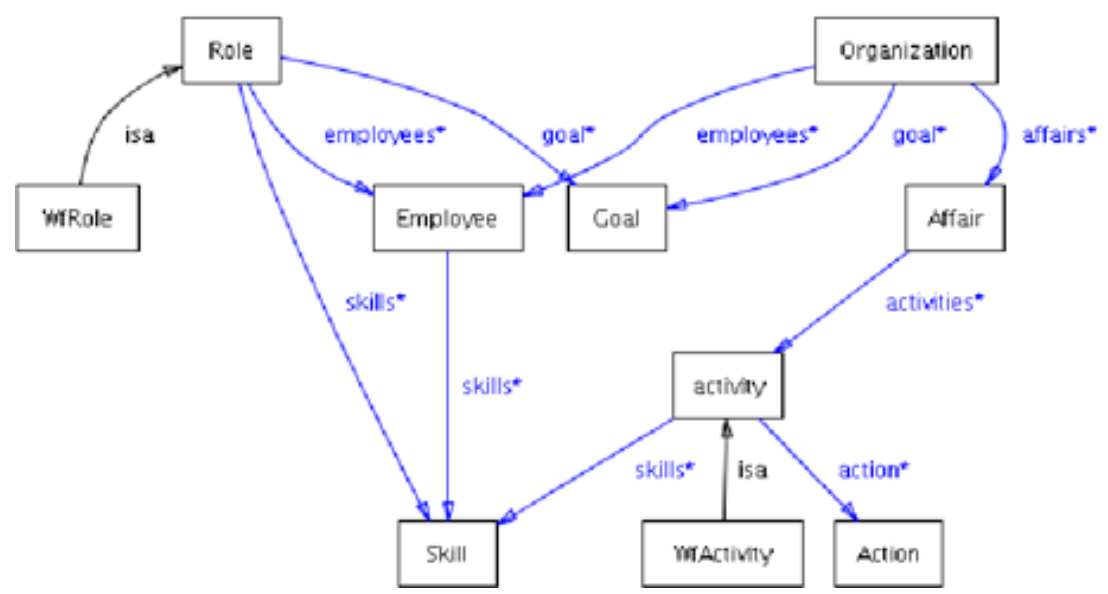

Fig. 2. Organizational Model of the Pellucid Framework 


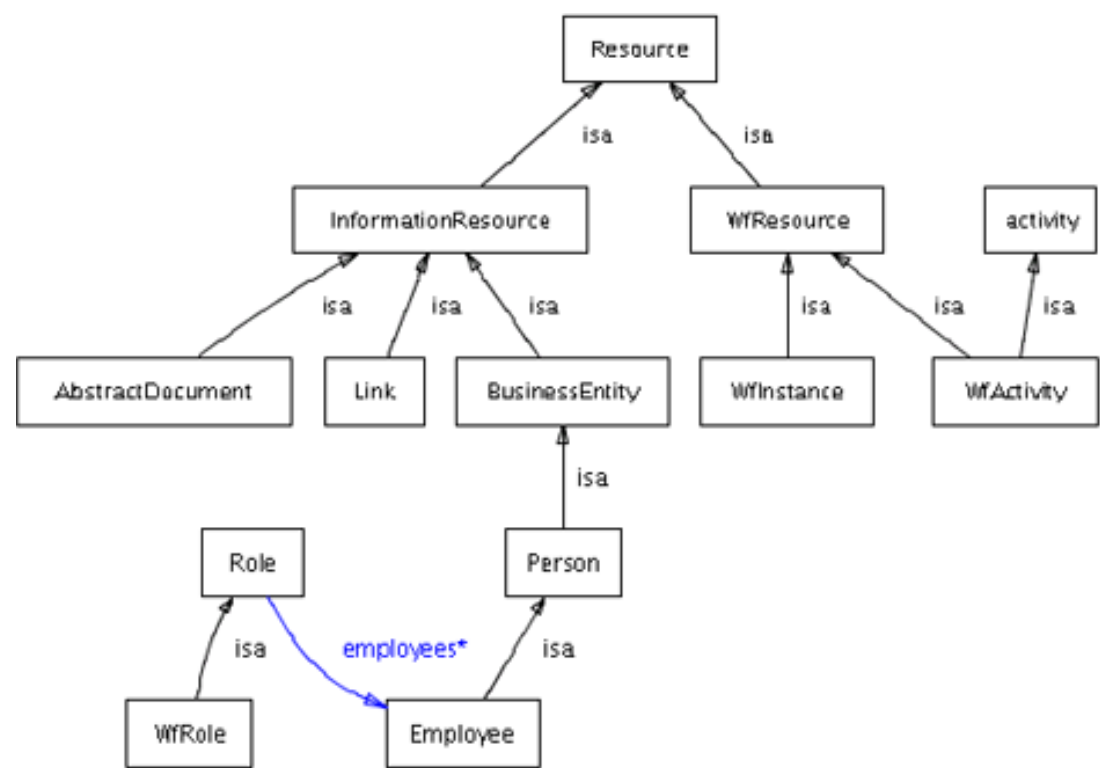

Fig. 3. Model of Resources in the Pellucid System

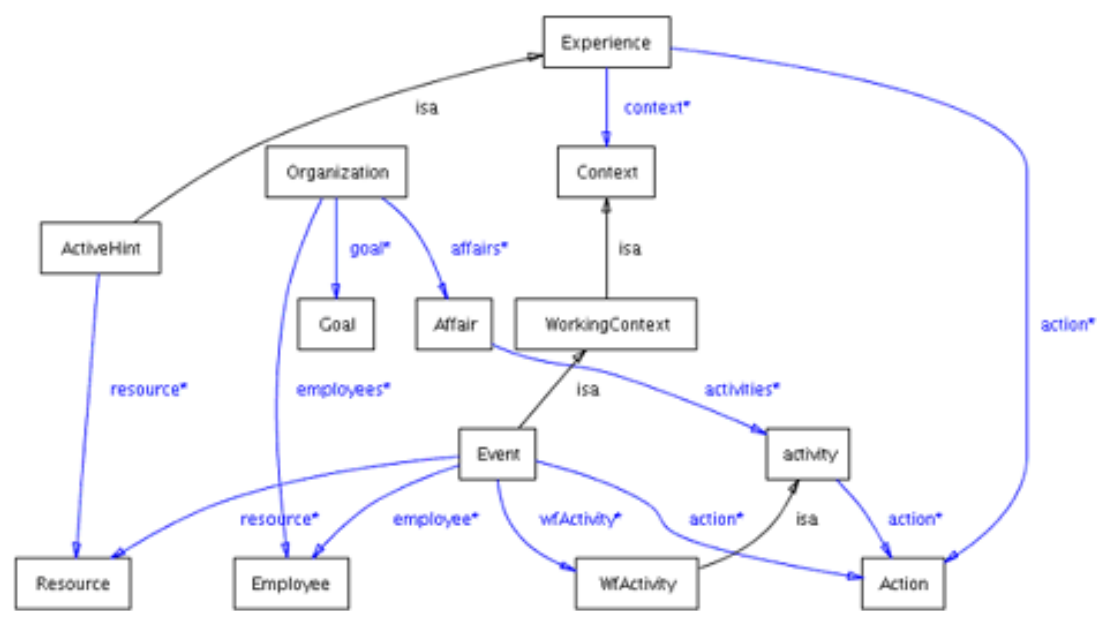

Fig. 4. Ontology Model in Pellucid

In scope of the Organizational Model we have also identified roles of the actors involved in organization together with their descriptions. As another important model all relevant resources in the organizations were modeled (Fig 3).

All the described ontologies create the generic ontology for any workflow driven application in public sector organizations. There are also three pilot sites 
were the Pellucid platform is installed. For all of these pilot sites a domainspecific ontology had to be created.

Active Hints $(\mathrm{AH})$ were created as examples of inferred knowledge. It was defined that $\mathrm{AH}$ is composed according to the following formula:

\section{$[$ ACTIVE_HINT $]=[$ WORK_CONTEXT $]+[$ ACTION $]+[$ RESOURCE $]+$ [EXPLANATION]}

The matching of the proper $\mathrm{AH}$ is then driven by the following condition:

\section{If [ACTIVE_HINT]. [WORK_CONTEXT] $=$ [CURRENT]. [WORK_CONTEXT] Then Display [ACTIVE_HINT]}

It is a simple rule which matches any $\mathrm{AH}$ from the $\mathrm{CM}$ which matches the users current work context. All the concepts from the formulas are modeled in the ontologies (see Fig. 3). Personal Assistant Agent was created to communicate the Active Hints returned from Pellucid to the end-user.

The ontology on Fig. 4 is the Design Model of the Pellucid platform according to the CommonKADS methodology. Eeach important ontology is included thus creating the overal model of the application domain.

In our framework we utilize DAML+OIL language for interfacing with existing systems, for interfacing among agents and also for processing of knowledge results. For system implementation we use the HP Jena library 12 which supports DAML+OIL and RDF models. Jena library also includes the RDQL query language and specialized query engine which is useful for reasoning and querying knowledge in the CM. We use JADE 1314] as the agent implementational platform.

\section{Conclusion}

Our experience management framework can be used not only for public administrational organizations, but also for other application areas. One of possible applications is military combat strategy recommendation system where the knowledge base is build from real world combat situations or virtual combat simulations. Context of the situation can be represented for example by geographical location of the combat troop. The recommendations can be transmitted to objects from satellites to wireless devices. Analogical research and development project is being prepared in conjunction with the Slovak Military Academy as a verification of our framework. Disease diagnosis and cure recommendation system is another application area this once from medical environment. In such case the context can be represented by patient's health condition, patient's physiological measures and patient's medical record. There are further possibilities for the exploitation of our framework including recommender system for consultation companies or help desk applications. In the future we plan to continue developing our framework for the above mentioned application areas. 


\section{References}

1. Goker, M. and T. Roth-Berghofer (1999). The development and utilization of casebased help-desk support system HOMER. Engineering Applications of Artificial Intelligence 12(6), 665-680.

2. Lacher S. and Koch M. (2000). An Agent-based Knowledge Management Framework.

3. Sarkar R. and Bandyopadhyay S. Developing an Intranet-based Knowledge Management Framework in a Consulting Firm: A Conceptual Model and its Implementation.

4. CoMMA project, http://www.si.fr.atosorigin.com/sophia/comma/

5. DECOR: Delivery of Context-Sensitive Organizational Knowledge, project outline at http://www.dfki.uni-kl.de/decor/deldec/D1-Final.pdf

6. Abecker, A., Bernardi, A., van Elst, L., Lauer, A., Maus, H., Schwarz, S., Sintek, M. (2001) "FRODO: A framework for distributed organizational memories. Milestone M1: Requirements analysis and system architecture", DFKI Document D-01-01, DFKI GmbH.

7. Abecker, A., Bernardi, A., Hinkelmann, K., Kühn, O., Sintek, M. "Context-aware, proactive delivery of task-specific knowledge: The KnowMore project", International Journal on Information Systems Frontiers, 2000, 2(3/4), pp. 139-162.

8. Pellucid - A Platform for Organisationally Mobile Public Employees, EU IST2001-34519 Project, 2002, http://www .sadiel.es/Europa/pellucid/.

9. M. Laclavik, Z. Balogh, L. Hluchy, G. T. Nguyen, I. Budinska, T. T. Dang: Pellucid Agent Architecture for Administration Based Processes, IAWTIC 2003, Vienna (2003).

10. R. Slota, M. Majewska, M. Dziewierz, K Krawczyk, M. Laclavik, Z. Balogh, L. Hluchy, J. Kitowski, S. Lambert: Ontology Assisted Access to Document Repositories for Public Sector Organizations. PPAM Conference (2003).

11. CommonKADS.

12. Jena (A Semantic Web Framework for Java) Homepage, http://jena.sourceforge.net/.

13. JADE (Java Agent DEvelopment Framework) Homepage, http://agentcities.cs.bath.ac.uk/docs/jade/

14. Giovani Caire (2002). JADE Tutorial Application-defined Content Languages and Ontol-ogy, http://jade.cselt.it/

15. DAML (DARPA Agent Markup Language) Homepage, http://www.daml.org/. 